\title{
Long-Term Safety of Tedizolid in a Patient With Spondilodiscitis After Switch From Linezolid Due to Toxicity
}

\author{
Rosa Maria Martínez Álvarez, MD, PhD, * Irene Navarro Pardo, BSc, $†$ \\ Estela Moreno García, MD, * and Rafael Huarte Lacunza, BSc ${ }^{\dagger}$
}

\begin{abstract}
The patient is a 57-year-old man with liver cirrhosis, Bricker anastomosis after a radical cystoprostatectomy and, a history of bacteremias caused by extended-spectrum $\beta$-lactamase-positive Escherichia coli, Enterococcus faecium, and Candida albicans. He presented with persistent low back pain and was diagnosed with vertebral osteomyelitis, for which he received ertapenem-linezolid treatment. However, after 20 days, linezolid had to be discontinued because of myelotoxicity and metabolic acidosis. The patient was switched to tedizolid, which, in combination with ertapenem, was successfully given for 114 days until biopsy showed no growth of gram-positive cocci. We conclude that tedizolid can be an alternative to linezolid in case of toxicity, especially in long-term treatments.
\end{abstract}

Key Words: tedizolid, linezolid, myelotoxicity,

long-term antibiotic treatment

(Infect Dis Clin Pract 2018;00: 00-00)

$\mathrm{T}$ edizolid phosphate is an inactive precursor (prodrug) of oxazolidinone phosphate, which is active against grampositive pathogens. It is approved for the treatment of acute bacterial skin and soft tissue infections caused by gram-positive micro-organisms, including methicillin-resistant Staphylococcus aureus. In addition, it showed in vitro activity against some atypical mycobacteria, atypical bacteria, and anaerobes. ${ }^{1}$

Linezolid, the first agent in the oxazolidinone group, has been associated with certain adverse effects such as anemia, thrombocytopenia, lactic acidosis, peripheral neuropathy, and optic neuropathy, often linked to prolonged treatments. In pivotal head-to-head clinical trials against linezolid, tedizolid was found to have noninferior efficacy and a better safety profile in terms of gastrointestinal adverse effects and platelet abnormalities. ${ }^{1,2}$ In a pooled safety data analysis from the two pivotal trials, ${ }^{3}$ a lower incidence of thrombocytopenia among patients who received tedizolid was observed, suggesting a potential clinical toxicity difference that the authors suggested should be confirmed in longer-term trials. In both pivotal studies, no serious adverse effects associated with long-term linezolid treatment, such as myelosuppression, peripheral or optic neuropathy, or lactic acidosis, were detected in tedizolid arms. It should be noted, however, that tedizolid treatment duration in these studies was only 6 days, which may have been not long enough to observe such effects. ${ }^{4}$

From the *Infectious Disease Unit, Internal Medicine Department; and $†$ Pharmacy Department, Miguel Servet University Hospital, Zaragoza, Spain. Correspondence to: Rosa Maria Martínez Álvarez, MD, PhD, Infectious

Diseases Unit, Antimicrobial Stewardship, Internal Medicine Department,

Miguel Servet University Hospital, Avenida Isabel La Católica 1-3, 50009

Zaragoza, Spain. E-mail: rmartineza@salud.aragon.es.

The authors have no conflicts of interest to disclose.

Editorial assistance was provided by Content Ed Net, Madrid, Spain.

Copyright $\mathbb{C} 2018$ Wolters Kluwer Health, Inc. All rights reserved.

ISSN: $1056-9103$
Here, we present a case of a patient with spondylodiscitis treated with tedizolid for a prolonged period after having discontinued linezolid because of hematological toxicity.

\section{CLINICAL CASE}

The patient is a 57-year-old man with a history of cryptogenic liver cirrhosis, Child-Pugh class $\mathrm{C}$, with esophageal varices and distal splenorenal shunt 8 years ago, awaiting liver transplantation. He was diagnosed with bladder neoplasia and underwent transurethral resection and subsequent radical cystoprostatectomy with urinary diversion (Bricker technique); the postoperative course was complicated by urologic sepsis with left pyelocaliceal ectasia that required urgent nephrostomy and admission to the intensive care unit for vasopressor support.

The intensive care unit stay was prolonged, with poor evolution complicated by bacteremias caused by extended-spectrum B-lactamase-positive Escherichia coli, Enterococo faecium, and Candida albicans, for which the patient received corresponding targeted treatment. Six months later, he presented with low back pain that had been lasting for a few weeks and could not be controlled with usual analgesia. The patient had no fever; two computed tomography scan images were compatible with vertebral osteomyelitis at D9-D10 and L3-L4 vertebrae level. Blood cultures were negative, and D9-D10 vertebral disc biopsy was performed but not processed because of technical problems. In view of the previous extended-spectrum $\beta$-lactamase-producing E. coli and E. faecium bacteremias, it was decided to treat the vertebral osteomyelitis with ertapenem and linezolid (requesting an authorization for off-label use) to target these micro-organisms as likely microbial agents.

In the first 20 days of antibiotic therapy, four units of red blood cell concentrate had to be transfused to treat persistent anemia without external bleeding: hemoglobin levels decreased from baseline $9.3 \mathrm{~g} / \mathrm{dL}$ to $7.8 \mathrm{~g} / \mathrm{dL}$ on day 6 of linezolid treatment, after which the first two units were transfused, and from $8.8 \mathrm{~g} / \mathrm{dL}$ on day 8 to $7.7 \mathrm{~g} / \mathrm{dL}$ on day 13 . The effect on the platelet count was less pronounced, with a transient decrease to 84.000 cells $/ \mu \mathrm{L}$, which quickly resolved and had no clinical consequences. Sodium bicarbonate was used to treat normal anion gap metabolic acidosis. Lactic acidosis was discarded; instead, these events were interpreted as possible adverse effects of linezolid treatment and urinary diversion in a patient with liver cirrhosis. Because of the hematological toxicity, we requested an authorization to substitute tedizolid $(200 \mathrm{mg} / 24 \mathrm{~h})$ for linezolid $(600 \mathrm{mg} / 12 \mathrm{~h})$. Although both linezolid and tedizolid had been reported to cause hematological adverse effects, we have opted for a switch to tedizolid based on the results of in vitro studies, tedizolid pharmacokinetic profile, and few case reports that suggested possible differences in mitochondrial toxicity and related adverse effects between the two drugs (discussed hereinafter).

The patient needed two units of red blood cell concentrate on the fourth day after the switch, after which the hematocrit level stayed stable at approximately $30 \%$ without further transfusions 
(Fig. 1). The dose of sodium bicarbonate could be reduced, although it could not be withdrawn until after several weeks after the switch because of persistent symptomatic acidosis. The tedizolid-ertapenem treatment was maintained for 114 days; at this point, although Gram stain of tissue biopsy showed presence of gram-positive cocci and yeast, the biopsy culture was positive only for $C$. albicans, for which a new targeted treatment was initiated.

\section{DISCUSSION}

Antibiotics of the oxazolidinone group, such as tedizolid and linezolid, inhibit bacterial protein synthesis by binding to the 50S subunit of the prokaryotic ribosome but may also inhibit mitochondrial protein synthesis due to the structural similarities between the prokaryotic and the mitochondrial ribosomes. Inhibition of mitochondrial ribosome is responsible for some adverse effects, such as myelosuppression, lactic acidosis, and peripheral and ocular neuropathy. Although most of these adverse effects are reversible, such toxicity may limit long-term use of oxazolidinone drugs. ${ }^{2}$

Besides, in 2011 the FDA issued a safety communication warning about possible serious CNS reactions associated with the concomitant use of linezolid and certain psychiatric medications. Both tedizolid and linezolid were found to be weak and reversible inhibitors of monoamine oxidase (a mechanism that may be behind the serotonergic syndrome). However, the higher antimicrobial potency and longer half-life of tedizolid allow for a single administration per day; besides, tedizolid's stronger binding to plasma proteins and resulting lower free drug concentration as well as minimal accumulation after several days of administration decrease the risk of interaction with monoamine oxidase, compared with linezolid. ${ }^{5,6}$

However, in vitro studies have shown that at therapeutic doses, tedizolid is a more potent inhibitor of mitochondrial protein synthesis than linezolid (dose- and time-dependent inhibition), which is not surprising, given that tedizolid has additional target binding sites. Flanagan et $\mathrm{al}^{7}$ found a correlation between increased linezolid trough concentrations $\left(\mathrm{C}_{\min }\right)$ and increased risk of adverse effects, which was linked to the prolonged maintenance of $\mathrm{C}_{\min }$ above the concentration necessary to inhibit $50 \%$ of mitochondrial protein synthesis $\left(\mathrm{IC}_{50}\right)$, without sufficient time for recovery of mitochondrial function between doses. In subsequent analyzes, the authors observed that tedizolid had faster dissociation and recovery from mitochondrial damage, compared with linezolid. The association between linezolid $\mathrm{C}_{\min }$ and mitochondrial toxicity also poses problems for prolonged treatments of multidrug-resistant tuberculosis. ${ }^{8}$

The pharmacokinetic profile of tedizolid, which permits once-daily administration of a therapeutic dose of $200 \mathrm{mg}$ (6 times less than the 1200-mg daily dose of linezolid), could explain such behavior. The time during which the free plasma concentration of tedizolid remains above the $\mathrm{IC}_{50}$ is decreased, allowing for the recovery of mitochondrial function between doses. ${ }^{2,9,10}$ The free plasma concentration of tedizolid $200 \mathrm{mg}$ administered every 24 hours is estimated to stay below the $\mathrm{IC}_{50}$ for 8 hours, whereas in case of linezolid $600 \mathrm{mg}$ given every 12 hours, the free plasma concentration of the drug is expected to be constantly above the $\mathrm{IC}_{50}$. Therefore, exposure to linezolid does not permit mitochondrial function recovery, thus increasing the toxicity. ${ }^{3}$

Although linezolid does not require dose adjustment in cases of hepatic or renal insufficiency, a greater risk of toxicity has been observed in patients with severe renal or hepatic insufficiency treated with linezolid. ${ }^{11}$ Tedizolid is not eliminated with urine, and although patients with renal or hepatic insufficiency were not included in the pivotal trials, no pharmacokinetics or safety profile issues were detected in patients with impaired renal function or in those requiring long-term hemodialysis during the phase 1 trials. ${ }^{3,10}$ In the phase 3 trials, where the maximum tedizolid treatment duration was 6 days, the reported incidences of peripheral neuropathy were similar in the two treatment arms $(1.2 \%$ and $0.6 \%$ for tedizolid phosphate and linezolid, respectively). In these pivotal studies, the effects on hematologic parameters were reported for platelets, neutrophils, and hemoglobin,
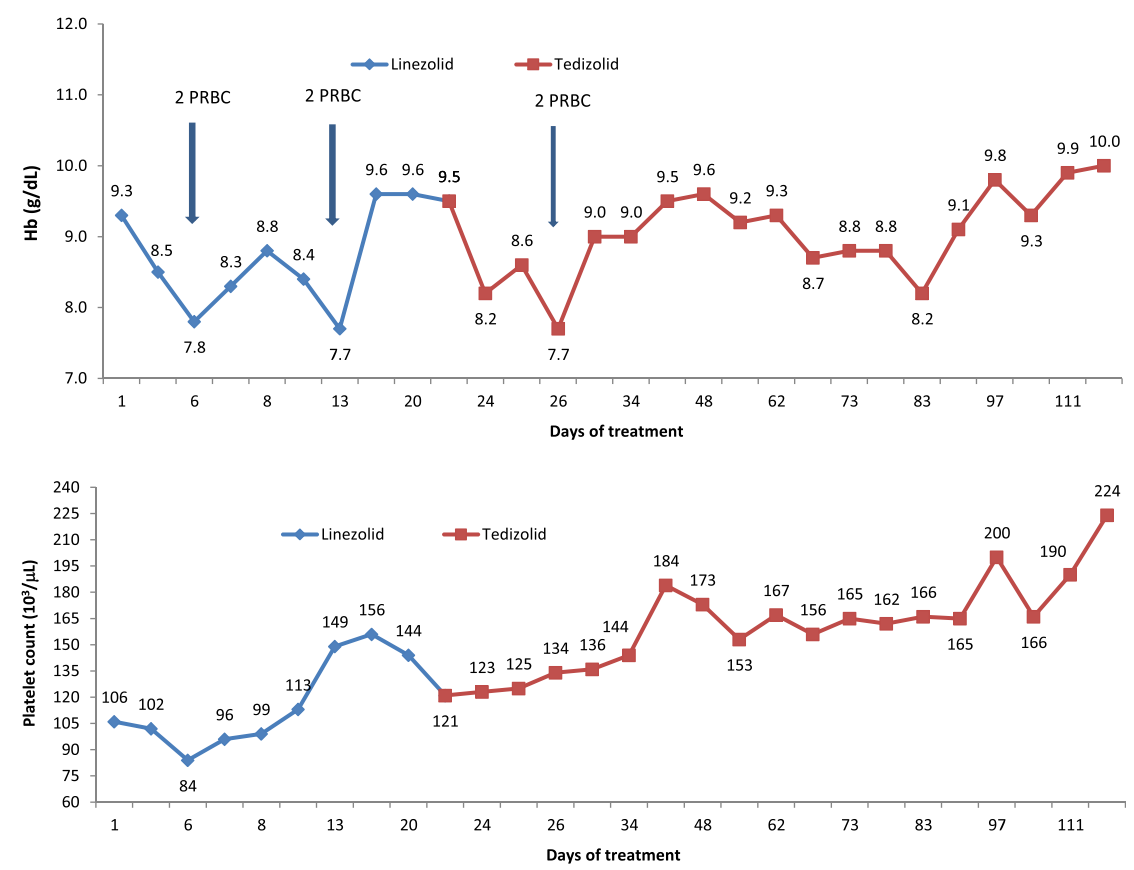

FIGURE 1. Hemoglobin levels and platelet count during linezolid (grey) and tedizolid (black) treatment. The switch to tedizolid was performed on day 23. The arrows indicate time points of red blood cell perfusions. PRBC, packed red blood cells. 
and the most notable differences between the treatment groups were observed for platelets. The percentages of patients with hemoglobin levels below the lower limit of normal on days 7 to 9 were $29.4 \%$ and $33.3 \%$ in the tedizolid and linezolid arms, respectively. ${ }^{12,13}$

There are few bibliographical references documenting the switch from linezolid to tedizolid due to toxicity. We reviewed the literature and found three articles describing long-term administration of tedizolid after linezolid-induced hematologic toxicity. ${ }^{10,14,15}$ These articles reported a total of four cases, in one of which tedizolid was given for 58 days and then withdrawn because of the decrease in hemoglobin levels, whereas in the other three cases, no toxicity issues were observed for the 56,88 , and 180 days of tedizolid treatment (the latter case was of a patient with multiple myeloma treated for nocardiosis).

In the case we describe, a transfusion was required in the first week after the switch from linezolid to tedizolid because of the low hemoglobin levels, but we attributed it to linezolid residual toxicity. The hematologic tolerance in our case allowed for a prolonged antibiotic treatment (114 days) without incidents. As for the acidosis, it is difficult to establish causality because it could be either an adverse drug reaction or a complication of the urinary diversion, given that metabolic acidosis occurs in $25 \%$ to $46 \%$ of patients with urinary diversion, depending on the technique used and the patient's renal function. ${ }^{16}$

Tedizolid may be considered as an alternative to linezolid in case of recognized toxicities associated with mitochondrial dysfunction and a need for a long-term treatment.

\section{REFERENCES}

1. Burdette SD, Trotman R. Tedizolid: the first once-daily oxazolidinone class antibiotic. Clin Infect Dis. 2015;61(8):1315-1321.

2. Flanagan S, McKee EE, Das D, et al. Nonclinical and pharmacokinetic assessments to evaluate the potential of tedizolid and linezolid to affect mitochondrial function. Antimicrob Agents Chemother. 2015;59(1): 178-185.

3. Lodise TP, Fang E, Minassian SL, et al. Platelet profile in patients with acute bacterial skin and skin structure infections receiving tedizolid or linezolid: findings from the Phase 3 ESTABLISH clinical trials. Antimicrob Agents Chemother. 2014;58(12):7198-7204

4. AEMPS. Informe de posicionamiento terapéutico PT-Tedizolid/V1/21102015. Available at: https://www.aemps.gob.es/
medicamentosUsoHumano/informesPublicos/docs/IPT-tedizolid-sivextro. pdf. Accessed June 22, 2017.

5. Flanagan S, Bartizal K, Minassian SL, et al. In vitro, in vivo, and clinical studies of tedizolid to assess the potential for peripheral or central monoamine oxidase interactions. Antimicrob Agents Chemother. 2013;57(7):3060-3066.

6. Urbina O, Ferrandez O, Espona M, et al. Potential role of tedizolid phosphate in the treatment of acute bacterial skin infections. Drug Des Devel Ther. 2013;7:243-265.

7. Flanagan SD, Dreskin H, Prokocimer P. Comparison of tedizolid and linezolid pharmacokinetics in Phase 1 studies [Abstract A-692]. Washington, DC: 54th Interscience Conference on Antimicrobial Agents and Chemotherapy; September 5-19; 2014.

8. Song T, Lee M, Jeon HS, et al. Linezolid trough concentrations correlate with mitochondrial toxicity-related adverse events in the treatment of chronic extensively drug-resistant tuberculosis. EBioMedicine. 2015;2(11): 1627-1633.

9. Fang E, Munoz KA, Prokocimer P. Characterization of neurologic and ophthalmologic safety of oral administration of tedizolid for up to 21 days in healthy volunteers. Am J Ther. 2017;24(2):e227-e233.

10. Yuste JR, Berto J, Del Pozo JL, et al. Prolonged use of tedizolid in a pulmonary non-tuberculous mycobacterial infection after linezolid-induced toxicity. J Antimicrob Chemother. 2017;72(2):625-628

11. Su E, Crowley K, Carcillo JA, et al. Linezolid and lactic acidosis: a role for lactate monitoring with long-term linezolid use in children. Pediatr Infect Dis J. 2011;30(9):804-806.

12. Moran GJ, Fang E, Corey GR, et al. Tedizolid for 6 days versus linezolid for 10 days for acute bacterial skin and skin-structure infections (ESTABLISH-2): a randomised, double-blind, phase 3, non-inferiority trial. Lancet Infect Dis. 2014;14(8):696-705.

13. Prokocimer P, De Anda C, Fang E, et al. Tedizolid phosphate vs linezolid for treatment of acute bacterial skin and skin structure infections: the ESTABLISH-1 randomized trial. JAMA. 2013;309(6):559-569.

14. Khatchatourian L, Le Bourgeois A, Asseray N, et al. Correction of myelotoxicity after switch of linezolid to tedizolid for prolonged treatments. J Antimicrob Chemother. 2017;72(7):2135-2136.

15. Matin A, Sharma S, Mathur P, et al. Myelosuppression-sparing treatment of central nervous system nocardiosis in a multiple myeloma patient utilizing a tedizolid-based regimen: a case report. Int J Antimicrob Agents. 2017;49(4):488-492.

16. Cano MGE. Complicaciones metabólicas y óseas de las derivaciones urinarias. Endocrinol Nutr. 2015;62(2):100-105. 